\title{
Critical coupling in plasmonic resonator arrays
}

\author{
Sinan Balci, ${ }^{*}$ Coskun Kocabas, and Atilla Aydinli \\ Department of Physics, Advanced Research Laboratories and Turk Telekom Laboratory, \\ Bilkent University, 06800 Ankara, Turkey \\ *Corresponding author: balci@fen.bilkent.edu.tr
}

Received April 29, 2011; revised June 13, 2011; accepted June 21, 2011; posted June 22, 2011 (Doc. ID 146572); published July 19, 2011

\begin{abstract}
We report critical coupling of electromagnetic waves to plasmonic cavity arrays fabricated on Moiré surfaces. Dark field plasmon microscopy imaging and polarization dependent spectroscopic reflection measurements reveal the critical coupling conditions of the cavities. The critical coupling conditions depend on the superperiod of the Moiré surface, which also defines the coupling between the cavities. Complete transfer of the incident power can be achieved for traveling wave plasmonic resonators, which have a relatively short superperiod. When the superperiod of the resonators increases, the coupled resonators become isolated standing wave resonators in which complete transfer of the incident power is not possible. Analytical and finite difference time domain calculations support the experimental observations. (c) 2011 Optical Society of America

OCIS codes: $240.6680,250.5403,050.2770$.
\end{abstract}

Jacobi's theorem in electrical engineering states that maximum power from an external source can be transferred to a load when the impedance of the load and the source are conjugates [1]. This theorem finds many implementations, especially when dealing with resonant circuits. It can be applied to analyze optical resonators such as Fabry-Perot cavities, microring resonators, and optical cavities [2,3]. The optimum condition to excite an optical cavity is achieved by matching the internal resonator loss to coupling loss, which is known as the critical coupling condition [2,3]. Here, we study the critical coupling of incident light to plasmonic cavity arrays. We find that complete transfer of energy can be achieved for a traveling wave plasmonic resonator at a critical metal film thickness. We provide an analytical expression based on a parametric model and experimental results to analyze the critical coupling in Moiré-type high- $Q$ plasmonic resonators.

Surface plasmon polaritons (SPPs) [4] show unique physical properties attracting great interest for a variety of applications [5-10]. Selective loading of the plasmonic surface with a metal or a dielectric thin film can result in a plasmonic cavity that provides lateral confinement along the propagation direction [5-10]. Localization and efficient coupling of the electromagnetic field in plasmonic cavities provides opportunities for new applications ranging from cavity quantum electrodynamics to nonlinear optics [11-13]. Metallic loss, however, limits the quality factor of the plasmonic cavities. A high- $Q$ plasmonic cavity can be achieved on a Moiré surface formed by superimposing two periodic patterns with slightly different periodicities [8].

The Moiré surfaces were fabricated using a holographic technique called interference lithography, the de-

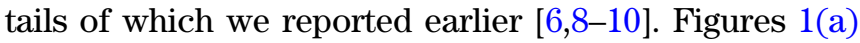
and 1(b) show atomic force microscope (AFM) images of the surfaces with superperiodicities of 9.0 and $2.5 \mu \mathrm{m}$, respectively. Figures $1(\mathrm{c})$ and $1(\mathrm{~d})$ show the crosssectional profiles of the surfaces. The gradual change in the profile is the essential feature to achieve high quality factors of around 150 .

To match the momentum of light to that of SPPs, the prism coupling technique in the Kretschmann geometry is used. SPPs are excited only when the projection of the wave vector of the incident light along the direction of propagation matches that of SPPs at the silver-air interface. The coupling of light to propagating surface plasmons depends strongly on the metal film thickness, which is extensively studied in the literature $[11,12]$. For example, Simon et al. showed that when an electromagnetic wave is incident on a flat Ag coated surface, Ag film thickness of either less than $30 \mathrm{~nm}$ or greater than $80 \mathrm{~nm}$ produces very shallow reflectivity minima [12].

We would now like to develop a parametric model that describes the conditions for the excitation of a plasmonic cavity. We describe the coupling of incident light to SPP cavities using a model [Fig. 2(a)] that was previously employed for optical resonators $[\underline{2}, 3]$. The equation for time evaluation of a cavity mode can be written as $[\underline{2}, \underline{3}]$

$$
\frac{d \psi}{d t}=\left(i \omega_{0}-\frac{1}{\tau_{0}}-\frac{1}{\tau_{1}}-\frac{1}{\tau_{2}}\right) \psi+\kappa s_{1+}+\kappa s_{2+},
$$

where $\psi$ is the amplitude of the SPP wave within the cavity, $\omega_{0}$ is the cavity resonant frequency, $1 / \tau_{0}$ is the decay rate due to the metallic loss, and $1 / \tau_{1}$ and $1 / \tau_{2}$ are decay rates of SPPs into the free space and the prism, respectively. Here, $\kappa$, the coupling coefficient between the incident light and the cavity, decays exponentially with the (a)

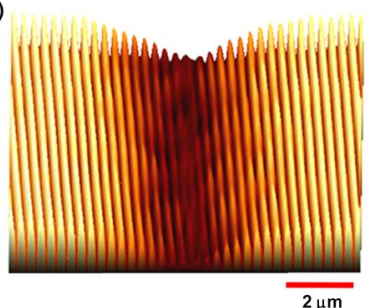

(c)

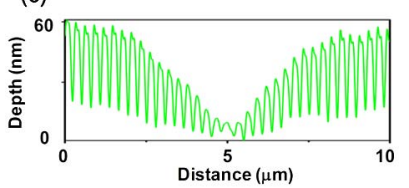

(b)

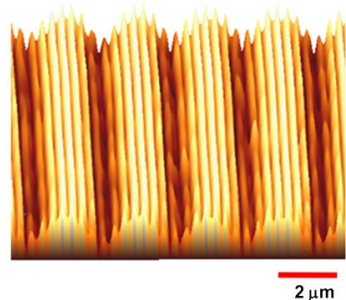

(d)

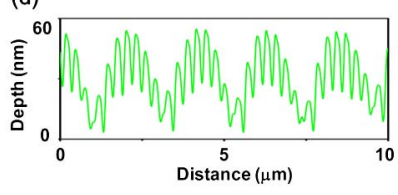

Fig. 1. (Color online) AFM images of Moiré surfaces with a superperiod of (a) 9.0 and (b) $2.5 \mu \mathrm{m}$. Line profiles extracted from (c) the AFM image in (a), and (d) the AFM image in (b). 
increase in the Ag film thickness. The decay rate into the prism is composed of decay rates in the forward and backward directions and satisfies the following relation: $\frac{1}{\tau_{f}}+\frac{1}{\tau_{b}}=\frac{2}{\tau_{2}}$. The derivative of amplitude of cavity mode with respect to time represents the evolution of the cavity mode in time. $s_{1+}$ and $s_{2+}$ are the incoming waves. We can derive the outgoing waves as $[2,3]$

$$
\begin{aligned}
& s_{1-}=e^{-i \beta L_{c}}\left(s_{2+}-\kappa^{*} \psi\right), \\
& s_{2-}=e^{-i \beta L_{c}}\left(s_{1+}-\kappa^{*} \psi\right),
\end{aligned}
$$

where $L_{c}$ is the resonator length and $\beta$ is the lateral component of the wave vector of the incoming wave. The coupling coefficient can be written in terms of the decay rates in the forward direction as $\kappa=\sqrt{\frac{1}{\tau_{f}}}$. The value of $\kappa$ can be calculated using perturbation analysis $[2,3]$. We choose only one incoming wave and one outgoing wave by setting $s_{2+}$ and $s_{1-}$ to zero. The reflection from the cavity has a Lorentzian form, which is written as

$$
\frac{s_{2-}}{s_{1+}}=e^{-i \beta L_{c}}\left(1-\frac{\frac{1}{\tau_{f}}}{i\left(\omega-\omega_{0}\right)+\frac{1}{\tau_{0}}+\frac{1}{\tau_{1}}+\frac{1}{\tau_{2}}}\right) .
$$

In order to reach the critical coupling at the resonance condition, the decay rate into the prism in the forward direction should be equal to the sum of the metallic loss $\left(1 / \tau_{0}\right)$, the decay rate of SPPs to free space $\left(1 / \tau_{1}\right)$, and the decay rate of SPPs to prism $\left(1 / \tau_{2}\right)$. This expression can be implemented and extended to various forms of plasmonic structures, such as a flat metal surface, a single plasmonic resonator, or coupled plasmonic resonators. The Moiré surface provides a flexible platform to tune the coupling between the cavities, resulting in different types of plasmonic resonators. A Moiré surface with a long superperiod behaves as an array of isolated plasmonic cavities. With a large separation between them, these cavities can be classified as standing wave resonators (SWRs). Ideally, this type of resonator merely has a standing wave without any power flow along the plane of the cavity. It may, however, decay out of the plane into both incoming and outgoing directions of the prism with equal and nonzero decay rates. For this type of configuration, complete transfer of energy to the cavity is not possible. Analytical calculation indicates that $75 \%$ of the input power at best can be transferred to the SWR [Fig. 2(b)]. On the other hand, a Moiré surface with a short superperiod forms travelling wave resonators (TWRs). This type of TWR has a nonzero group velocity and a net flow of electromagnetic power. The time evaluation of the mode in the $n$th cavity is written as [2,, 3$]$

$$
\begin{aligned}
\frac{d \psi_{n}}{d t}= & \left(i \omega_{n}-\frac{1}{\tau_{0}}-\frac{1}{\tau_{1}}-\frac{1}{\tau_{2}}\right) \psi_{n}+i \kappa_{1} \psi_{n+1}+i \kappa_{1} \psi_{n-1} \\
& +\kappa s_{1+}+\kappa s_{2+},
\end{aligned}
$$

where $\kappa_{1}$ is the coupling coefficient between the cavities and $\omega_{n}$ is the resonance frequency of a cavity. This difference equation has a traveling wave solution, and
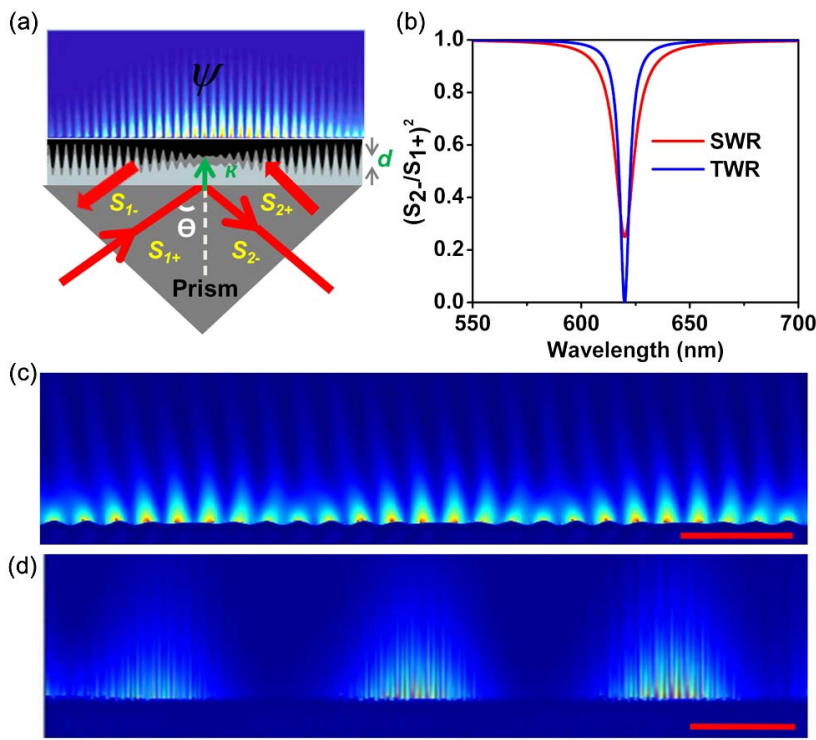

Fig. 2. (Color online) (a) Kretschmann configuration used for coupling of light to SPPs where $d$ is the Ag film thickness. (b) Analytically calculated reflection curves under coupling conditions for an SWR and a TWR. FDTD calculated SPP electric field distribution for (c) 2.5 and (d) $15.0 \mu \mathrm{m}$ coupled plasmonic resonator arrays indicating TWR and SWR properties, respectively. The scale bars are (c) 1 and (d) $5 \mu \mathrm{m}$ long. The vertical length of images in (c) and (d) is $\sim 1 \mu \mathrm{m}$ long.

therefore complete extinction of reflectivity is possible, resulting in full transfer of the input power to the SPP cavities [Fig. 2(b)]. Finite difference time domain (FDTD) calculated electric field distribution in cavities supporting the analytical results is given in Figs. $\underline{2(\mathrm{c})}$ and $\underline{2(\mathrm{~d})}$, respectively.

We experimentally observed critical coupling in plasmonic cavities using a technique called dark field plasmon microscopy (DFPM) $[13,14]$ and reflection measurements. Figures 3(a)-3(d) show FDTD calculated electric field distribution of cavities with varying Ag film thicknesses. The field inside the cavity reaches its maximum value at a thickness of $\sim 30 \mathrm{~nm}$. To image the field distribution, we used DFPM, providing the image of
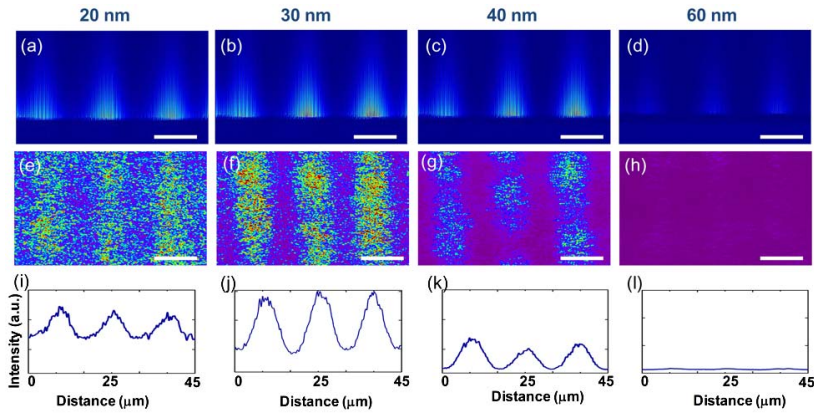

Fig. 3. (Color online) FDTD calculated two-dimensional electric field distribution at the cavity wavelength for $15.0 \mu \mathrm{m}$ long plasmonic resonators with Ag film thickness of (a) 20, (b) 30, (c) 40, and (d) $60 \mathrm{~nm}$. The vertical length of images in (a)-(d) is $\sim 1 \mu \mathrm{m}$ long. DFPM images of coupled resonators at the resonance wavelength for Ag film thicknesses of (e) 20, (f) 30 , (g) 40 , and (h) $60 \mathrm{~nm}$. The white bars indicate a $10 \mu \mathrm{m}$ long distance. Line scans perpendicular to the long axis of plasmonic resonator arrays with Ag film thicknesses of (i) 20, (j) 30, (k) 40, and (l) $60 \mathrm{~nm}$. 

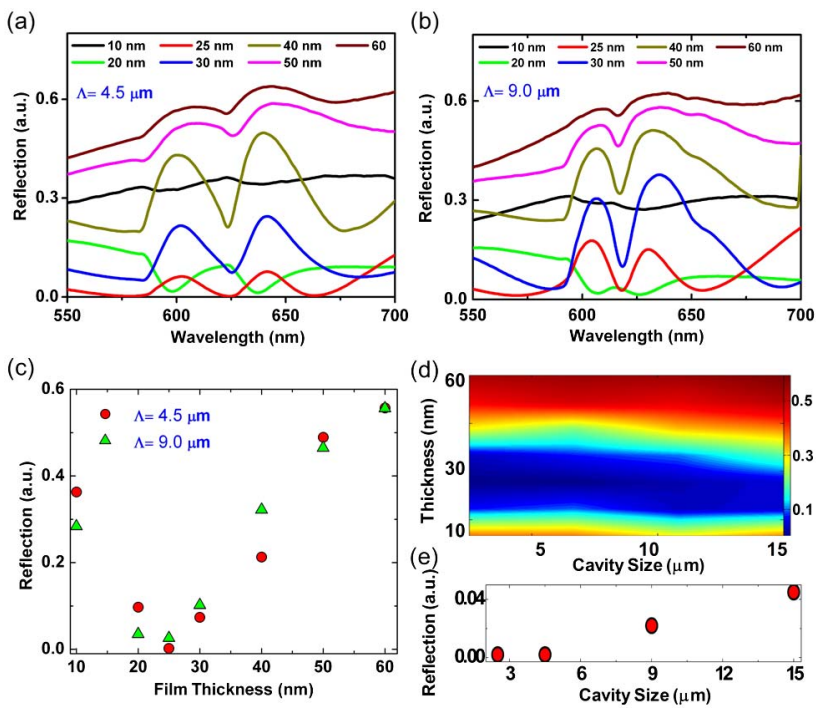

Fig. 4. (Color online) Reflection curves from Moiré surfaces containing (a) 4.5 and (b) $9.0 \mu \mathrm{m}$ long SPP cavities. The reflection curves have been obtained at an incidence angle of $42.5^{\circ}$, where the maximum coupling of incident light to SPPs can be achieved for a given Ag film thickness. It should be noted that reflection from the band edges also goes to zero under critical coupling in (a). (c) Using the reflection curves in (a) and (b), minima of the reflection curves have been drawn. At $25 \mathrm{~nm} \mathrm{Ag}$ film thickness, reflection from the SPP cavities reaches a minimum value. (d) Reflection from the cavity mode calculated from the dispersion curves for cavity sizes of $2.5,4.5,9.0$, and $15.0 \mu \mathrm{m}$ with varying $\mathrm{Ag}$ film thicknesses from 10 to $60 \mathrm{~nm}$. (e) The reflection from the cavity mode at a $\mathrm{Ag}$ film thickness of $25 \mathrm{~nm}$ increases with the increase in cavity size.

the intensity profile of the plasmonic cavity mode. DFPM has the advantage that during imaging of plasmonic resonators, the intensity of the scattered light does not change with time as in the case of emission of dye molecules. The cavities are excited with a tunable supercontinuum laser (Koheras, Denmark) through a glass prism. The scattered SPP waves are collected in the far field with a microscope objective and imaged on a CCD camera. The DFPM images are shown in Figs. 3(e)-3(h). The cross-sectional profiles of the images are given in Figs. 3(i)-3(1). As expected, the field intensity in the cavity shows a maximum at critical film thickness.

Furthermore, we characterized the fabricated cavities in detail by spectroscopic reflection measurements. Dispersion curves have been obtained as a function of Ag film thickness.Figures 4(a) and 4(b) show the reflectivity curves for superperiods of $\overline{4.5}$ and $9.0 \mu \mathrm{m}$. The superperiodicity of the surface profile determines the cavity size as well as the distance between the cavities, which defines the coupling between the cavities. The dip at $620 \mathrm{~nm}$ is due to the cavity state. As we increase the film thickness, coupling to the cavity state increases up to a thickness of $25 \mathrm{~nm}$ and then starts to decrease [15]. Figure 4(c) shows the thickness dependence of the reflection at the resonance wavelength. At the critical film thickness, reflection at the cavity state goes down to zero, which indicates a complete transfer of the incident energy to the cavity. As predicted by the analytical model, all of the incident optical power can be transferred to cavities that form TWRs. The coupling coefficient between the cavities defines the tuning parameter between TWR and SWR. Figures 4(d) and 4(e) show the reflectivity at the resonance as a function of both superperiod and film thickness. These experimental observations agree very well with analytical and FDTD calculations.

In conclusion, we have demonstrated critical coupling of electromagnetic waves to plasmonic resonator arrays. The DFPM imaging and polarization dependent spectroscopic reflection measurements reveal the critical coupling conditions of the cavities. A complete transfer of the incident power can be achieved for TWRs having relatively short superperiods. As the superperiod increases, the coupled resonators become isolated SWRs where the complete transfer of the incident power is not possible. The results are promising in applications such as plasmonic lasers, surface enhanced optics, and spectroscopy because of the high electric field intensity generated under critical coupling conditions.

This work received financial support from the Scientific and Technological Research Council of Turkey (TUBITAK) (110T790) and Unam-Regpot (203953). S.B. acknowledges TUBITAK.

\section{References}

1. P. Horowitz and W. Hill, The Arts of Electronics (Cambridge University, 1989).

2. C. Manolatou, M. J. Khan, S. Fan, P. R. Villeneuve, H. A. Haus, and J. D. Joannopoulos, IEEE J. Quantum Electron. 35, 1322 (1999).

3. A. Yariv, Electron. Lett. 36, 321 (2000).

4. H. Raether, Surface Plasmons (Springer-Verlag, 1986).

5. E. Cubukcu, E. A. Kort, K. B. Crozier, and F. Capasso, Appl. Phys. Lett. 89, 093120 (2006).

6. S. Balci, A. Kocabas, C. Kocabas, and A. Aydinli, Appl. Phys. Lett. 98, 031101 (2011)

7. J.-C. Weeber, A. Bouhelier, G. Colas des Francs, S. Massenot, J. Grandidier, L. Markey, and A. Dereux, Phys. Rev. B 76, 113405 (2007).

8. A. Kocabas, S. S. Senlik, and A. Aydinli, Phys. Rev. Lett. 102, 063901 (2009).

9. S. Balci, A. Kocabas, C. Kocabas, and A. Aydinli, Appl. Phys. Lett. 97, 131103 (2010).

10. S. Balci, M. Karabiyik, A. Kocabas, C. Kocabas, and A. Aydinli, Plasmonics 5, 429 (2010).

11. M. N. Zervas, Opt. Lett. 16, 720 (1991).

12. H. J. Simon, D. E. Mitchell, and J. G. Watson, Am. J. Phys. 43, 630 (1975).

13. H. Ditlbacher, A. Hohenau, D. Wagner, U. Kreibig, M. Rogers, F. Hofer, F. R. Aussenegg, and J. Krenn, Phys. Rev. Lett. 95, 257403 (2005).

14. L. Helden, E. Eremina, N. Riefler, C. Hertlein, C. Bechinger, Y. Eremin, and T. Wriedt, Appl. Opt. 45, 7299 (2006).

15. P. Andrew and W. L. Barnes, Science 306, 1002 (2004). 